\title{
Chondroblastoma of the Patella with Secondary Aneurysmal Bone Cyst, an Easily Misdiagnosed Bone Tumor: A Case Report with Literature Review
}

Jianping Zheng

General Hospital https://orcid.org/0000-0003-0086-0935

Ningkui Niu

General Hospital

Jiandang Shi ( $\sim$ Jiandang-Shi@outlook.com )

General Hospital https://orcid.org/0000-0002-3530-1167

\section{Xu Zhang}

General Hospital

Xi Zhu

General Hospital

Jiali Wang

General Hospital

Changhao Liu

General Hospital

\section{Case report}

Keywords: Chondroblastoma, patella, aneurysmal bone cyst, misdiagnosed, biopsy

Posted Date: September 24th, 2020

DOI: https://doi.org/10.21203/rs.3.rs-80091/v1

License: (c) (1) This work is licensed under a Creative Commons Attribution 4.0 International License.

Read Full License 


\section{Abstract}

Background: Chondroblastoma (CB) is a rare, primary, benign bone tumor that common affects men aged 15-20 years. It is usually detected in the epiphysis of the long bones, such as the proximal femur, humerus, and tibia. However, $\mathrm{CB}$ is extremely rare in the patella, especially in combination with secondary aneurysmal bone cyst (ABC). In fact, this complicated condition can be easily confused with other common bone tumors of the patella.

Case presentation: We have presented here the case of a 30-year-old man who was suffering from the anterior knee pain for the past 6 months that got aggravated since the past 2 weeks. Osteolytic bone destruction in the patella could be detected in both his X-ray and computed tomography (CT) examinations, while the magnetic resonance imaging (MRI) detected a fluid level. Accordingly, secondary $A B C$ was presumed. We diagnosed the condition as giant cell tumor (GCT) with secondary $A B C$ and accordingly performed curettage inside the focus region by autogenous bone grafting in accordance with the patient's medical history, physical manifestations, results of physical and ancillary examinations, and the disease characteristics. However, the in- and postsurgical outcomes indicated that the patient's histopathology was consistent with that of typical $C B$, suggesting a definitive error in diagnosis. Accordingly, the patient was finally diagnosed with patella $C B$ along with secondary $A B C$.

Conclusions: Past studies have demonstrated that the 3 commonest bone tumors affecting the patella are GCT, CB, and ABC. We believe that the condition of patella GCT and CB accompanied with secondary $A B C$ can be easily misdiagnosed. Performing incision biopsy or excision biopsy and conducting histological examination may be the most effective approach to identify and distinguish among these diseases.

\section{Background}

Chondroblastoma (CB) is a rare, primary, benign bone tumor that originates from the cartilages, accounting for only $1-2 \%$ of all primary bone tumor cases reported for the whole body ${ }^{[1]}$. CB arises from the secondary ossification center of the epiphysis and the epiphyseal plate, and it is common in older children and adolescents, but rare in adults. The ratio of $\mathrm{CB}$ occurrence between men and women is approximately 3:2. This type of tumors often affect the proximal epiphysis of the femur, humerus, and tibia ${ }^{[2,3]}$, while those affecting the patella are extremely rare ${ }^{[4,5]}$. Only a few cases of CB combined with secondary $A B C^{[6]}$ have been reported until date, and they can be easily misdiagnosed as giant cell tumor (GCT) or ABC. Very few cases of $C B$ combined with secondary ABC have been reported previously, with the main focus being the treatment regime, but not with respect to the differential diagnosis with GCT or $A B C$. The present article reports a patient who was diagnosed as patella $C B$ with secondary $A B C$ and admitted to our hospital; we have presented here a review of his diagnosis and the potential differential diagnosis.

\section{Case Presentation}


A 30-year-old man presented with the complain of pain in his left knee for half a year that had aggravated since the past 2 weeks. There was no obvious inducement of the knee pain 6 months ago, and, although it was aggravated after activities, the pain remained tolerable. The patient did not seek any treatment because the pain did not affect the patient's daily life. However, when the patient felt increased pain since past 2 weeks, he sought medical help from the hospital.

His physical examination revealed obvious tenderness in the left anterior patella, no swelling in the left knee, and no effusion in the joint cavity. Normal color and temperature of the local skin, negative result on the floating patella test, and no knee instability and locking were noted. He showed normal knee movement. No abnormality in the laboratory test results were recorded before surgery.

Imaging examination included anteroposterior and lateral X-ray before surgery. The density of the left patella was found to decrease uniformly and the joint space was normal. Presurgerical CT revealed osteolytic cystic bone destruction at the middle and lower portions of the left patella, in a lobulated or worm-eaten shape with sharp edges. The local bone cortex had thinned. The focus boundary was clear, and there was no sign of sclerosis at its edge. Presurgical MRI revealed abnormal MRI signals of patchy shadow with clear boundary in the left patella. The T1-weighted image showed hypointensity, while the T2-weighted image showed hyperintensity. The fluid level could be observed on the sagittal or axial view of the T2-weighted image, suggestive of $A B C$ as a concern. Presurgical lung CT revealed no neoplasm metastasis focus (Fig. 1).

The condition could be diagnosed as GCT with secondary ABC (Enneking stage III) based on the patient's age and imaging findings. The surgery was performed after completing the presurgical preparations. However, no needle biopsy was performed before the surgery. Intra-operative freezing of incision biopsy was however conducted to further confirm the diagnosis. The specific reasons for the same were as follows:

1. The patient's age and imaging findings were consistent with those of GCT and, most importantly, GCT was partially accompanied by secondary $A B C$, making presurgical diagnosis credible.

2. For commonly used fine-needle aspiration and core needle biopsy, only a few sample types could be collected due to restriction by several factors, including the type and size of the focus as well as the sample-collecting experience of the operator. However, some patients showed a negative biopsy result, which makes incision biopsy mandatory for the final confirmation of the diagnosis.

3. The $A B C$ manifestation and the small focus identified in the pre-surgery MRI raised difficulty in collecting sufficient samples for aspiration and needle biopsy.

The surgical process involved administration of anesthesia, after which the patient was made to lie in a supine position, and his left lower limb and right ilium were disinfected and draped. First, a 6-cm long longitudinal incision was made at the left patella. Then, fenestration was performed with an osteotome on the anterior patella after it came out. The size of the cortical window was the same as that of the focus. An oval shape was drilled on the patella to enable uniform distribution of the stress on the cortical window. Fresh blood was oozing out of the focus after the fenestration, and the focus had a multi- 
dividing shape with some myxoid substance inside it. The cartilage surface of the patella was relatively complete. Once the focus tissue was dispatched for intra-operative freezing biopsy, the focus content was removed completely with a curette. Then, the partition inside the focus was cleared by using a highspeed burr. Finally, the boundary of the focus was expanded along its periphery. After well cleaning of the focus, the bleeding was stopped and the wound was repeatedly rinsed with povidone iodine solution and isotonic saline. Autologous iliac bone was next harvested from the contralateral one and grafted at the focus (Fig. 2), followed by suturing of the incisions in turn.

The left knee joint was reviewed after the surgery, and the grafted bone at the focus was found to have a good effect on the anteroposterior and lateral films and on 3D-reconstructed CT (Fig. 3). The static quadriceps exercise was started immediately after the surgery. The knee joint was next fixed with braces at the extension position for 4 weeks, after which the functional exercise for the knee was started.

The reports for the intra-operative freezing and post-surgery pathological outcomes revealed that the tumor tissues in the left patella were composed of osteoclast-like multi-nucleated giant cells and chondroid matrix. Accordingly, CB was considered (Fig. 4).

\section{Discussion}

$\mathrm{CB}$ is a benign bone tumor that common affects the epiphysis of the long bones. The average onset age of $C B$ is $15-20$ years; its local recurrence rate is approximately $15 \%{ }^{[7]}$. Kolodny et al. reported CB in 1927 and described it as GCT-containing cartilage ${ }^{[8]}$. This disease was also depicted as a cartilage-like GCT [9] in the epiphysis or as calcified GCT ${ }^{[10]}$ before it was officially named CB in the year $1942{ }^{[11]}$. Hence, there is extensive overlap between CB and GCT in some aspects, which makes it critical to establish a system for accurately diagnosing this disease in order to design optimized treatment plan.

According to the literature, 3 bone tumors commonly affect the patella, namely, GCT, CB, and ABC [14, 15] Among these, GCT is the commonest, accounting for $33 \%$ of all patella bone tumor cases, followed by $\mathrm{CB}$ and $A B C$ at $16 \%$ and $5 \%$, respectively. However, secondary $A B C$ is further observed in $C B$ and $G C T$ [12, 14$]$, which adds to the difficulty of accurately diagnosing such a complex disease. Laitinen et al. claimed that ${ }^{[15]} \mathrm{CB}, \mathrm{GCT}$, and $\mathrm{ABC}$ share similar clinical manifestations and imaging characteristics. Until date, no studies have reported the manner of distinguishing among these diseases as the rare related publications have mainly focused on the treatment of $\mathrm{CB}$. This is first article to have regarded misdiagnoses as the starting point of research while elaborating the process through which $C B$ with secondary $A B C$ is misdiagnosed as GCT with secondary ABC, and the other common bone tumors. We have also put forward an effective manner to identify the abovementioned diseases by reviewing similar literature published before and have provided a detailed overview of differences and similarities among the 3 commonest patella bone tumors to create a theoretical basis for their accurate diagnoses.

In terms of incidence of characteristics, $\mathrm{CB}$ often affects the proximal epiphysis of the long bones, mostly in the femur, humerus, and tibia. The average onset age of CB patients is 15-20 years, with more number 
of male patients affected. GCT is commoner in people in their thirties, generally in the age range of 2040 years. In addition, this disease is common in the epiphysis of the long bones, mostly in the distal femur, proximal tibia, and distal radius ${ }^{[16]}$, and it involves slightly more number of female patients. There are two types of $A B C$ : primary and secondary ${ }^{[17]}$. The onset age for primary $A B C$ is generally $<20$ years (mostly between 10 and 20 years) and it is located in the epiphysis of the long bones (such as the femur and tibia), with the involvement of more number of female patients ${ }^{[18]}$. The latter one is often $A B C$ secondary to GCT, CB, telangiectatic osteosarcoma, and osteoblastoma ${ }^{[22]}$. All of the abovementioned 3 tumor types occur rarely in the patella. The present patient manifested highly similar features to those of $A B C$ secondary to GCT, based on the onset characteristics of the common patella tumors. This aspect overemphasizes the importance of onset characteristics in the diagnostics that often leads to misdiagnosis.

There are no specific differences in the clinical presentation of the 3 diseases, as the physical examination and laboratory tests for all 3 diseases feature knee pain without or with knee swelling or redness. In addition, a light tenderness of the knee was noted in the physical examination, albeit the knee joint movement was basically normal. Most patients obtained normal laboratory test results, such as for the erythrocyte sedimentation rate, C-reactive protein, and alkaline phosphatase. The present patient only showed knee pain, with the detection of only knee tenderness in his physical examination. Therefore, his condition could not be further identified from the perspective of clinical manifestations alone.

According to the imaging features of this disease, CB manifests as a radioparent lesion in the X-ray with a clear and sclerotic border. Its bone cortex becomes thin, and lobulated ridge can be seen inside. On CT, it is presented as a divided osteolytic lesion with sclerotic boundary and calcification scattering inside the focus. On MRI, the lesion manifests as a lobulated lesion with a clear boundary, showing iso/hypointensity on T1-W1 images and mixed intensity or hyperintensity focus on T2-W1 images. The fluid level can be seen in case of secondary $A B C{ }^{[4,6]}$. CB was featured as an integrated sclerotic bone at the margin of focus and scattered calcified lesion, wherein the focus could extend to the subchondral bone, although it seldom entered the joint space ${ }^{[19]}$. GCT also presented on X-ray images and CT as an osteolytic lesion. Soap-bubble-like changes could be seen in some lesions, which led to the destruction of the bone. On MRI, T1-W1 showed hypointensity and T2-W1 showed hyperintensity focus with no obvious specificity. As GCT is invasive to a certain extent, it is often complicated by pathological fractures. Occasionally, it even breaks through the cortical bone and invades the nearby tissues, inducing pulmonary metastasis in some patients. The appearance of multi-chamber lesions suggest secondary $A B C{ }^{[4,20]}$. $A B C$ is featured as multi-chambers, expansible, osteolytic lesions, with thinner bone cortex on CT and MRI, with the fluid level and focus separation noted on MRI ${ }^{[17,21]}$. This patient demonstrated lobulated osteolytic lesion with a clear boundary on images, although no calcification was noted on the focus margin or in the focus, which is inconsistent with the characteristics of CB reported until date. On $\mathrm{CT}$, the focus was found to be connected with the knee joint at the junction between the inferior patella and the articular surface. This trait is consistent with the imaging findings of GCT with invasiveness to a certain extent. Further observation of the patient's knee MRI revealed that the fluid level and multiple 
partition exists in the focus; these features are highly consistent with those of the manifestations of $A B C$. In consideration of the abovementioned imaging findings, the onset characteristics of GCT and the observation of fresh blood oozing out of the focus during the surgery helped indicate a possible diagnosis of GCT complicated by ABC. However, it is clear that an accurate diagnosis of bone tumor cannot rely only on the onset characteristics of the disease, clinical features, and common imaging findings.

The patient's post-surgery histology revealed that the tumor comprised of confluent proliferating cells in round or oval shapes under low-power microscope. In addition, osteoclast-like multi-nucleated giant cells were distributed in the entire tumor region. Under high-power microscope, the tumor cells appeared round in shape with clear borders. Their cytoplasm was light red in color or transparent with nuclear groove. Pink cartilage-like matrix was also noted. All of these features are highly consistent with those of the specific manifestations ${ }^{[4,5,6,23]}$ (proliferated chondroblasts observed in the laminar regions, accompanied by cartilage matrix and some multi-nucleated cells. Calcification may also appear in some areas) of CB. Walled cystic area that appeared in the fibrous septa or some multi-nucleated giant cells contained blood and involved immature bone formation. The diagnosis of $A B C$ for this patient was confirmed with reference to both the pre-surgery MRI findings and those observed during the surgery. The histology of GCT presented with numerous giant cells, short spindle cells, calcified bone tissues, and some mitotic figures. The invasive GCT, on the other hand, manifested as concentrated bone matrix

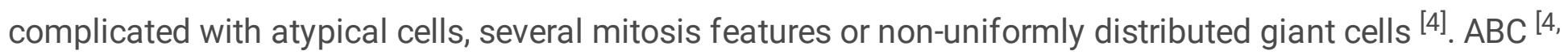
${ }^{24]}$ is characterized by empty blood-containing cavities or red cells. The cavity is separated by fibrous connective tissues composed of spindle cells, huge multinucleate cells, macrophages, hemosiderosis, and trabecular bones. Therefore, it can be concluded that the histological diagnosis is essential to identify tumors, but the prerequisite for obtaining an accurate pathological diagnosis involves the selection of an appropriate biopsy technique.

Currently, the frequently used biopsy technologies mainly include fine-needle aspiration, core needle biopsy, incision biopsy, and excision biopsy. Owing to the involvement of small trauma, less complications, lower cost, and ease of operation, the first two technologies are favored by bone oncologists ${ }^{[25,26]}$. However, there are several other factors that affect the diagnosis rate with the use of these two approaches. For fine-needle aspiration biopsy, mainly cell samples are collected. Although this process can be improved by collecting samples from various parts, the accuracy rate for identifying $A B C$ is approximately $70 \%$ with this technology. Such a low accuracy rate may be attributed to the atypical cytological features of $A B C$, which makes fine-needle aspiration biopsy not recommendable for presurgery diagnosis of patients with $\mathrm{ABC}$. The samples collected for core needle biopsy mainly include cells and tissues, which help improve the diagnosis rate to $81 \%$, also because it is associated with the focus size. For focus size $\geq 3 \mathrm{~cm}$, the diagnosis rate can reach $89.2 \%$, but for focus size $<3 \mathrm{~cm}$, the rate can only reach $73.4 \%{ }^{[27,28]}$. Incision biopsy has been regarded as the gold standard for diagnosis because of its good sample-collection system and high diagnosis rate. Considering that outmost care was ensured during imaging (showing secondary aneurysmal bone cyst [ABC] with a small focus) of this patient 
before the surgery and at the onset of characteristic development, incision biopsy was adopted instead of pre-surgery needle biopsy.

In case of insufficient tissues available for biopsy or in case of a lack of chondrogenic differentiation area in the limited samples, monitoring the K36M mutation on $H 3 F 3 A$ and $H 3 F 3 B$ by immunohistochemistry or polymerase chain reactions is an effective technique. Past studies have shown that ${ }^{[29,30]}$ histone $\mathrm{H} 3.3$ mutations exist in both GCT and CB, mainly H3F3A mutations in GCT and H3F3B mutations in $\mathrm{CB}$. This feature has encouraged the development of new diagnostic tools ${ }^{[31,32]}$ that uses the gene sequencing for $\mathrm{H} 3 \mathrm{~F} 3 \mathrm{~A}$ and $\mathrm{H} 3 \mathrm{~F} 3 \mathrm{~B}$ or uses the specific antibodies of G34W and K36M mutation for immunohistochemical analysis to enable distinguishing between CB and GCT. However, the wide application of these tools in the clinical practice can only be realized after a long time.

Although this patient was not diagnosed correctly before the surgery, his benign bone tumor remained at the Enneking stage III. Therefore, in accordance with the conventional treatment approach adopted by the current practices, the patient's focus can be aggressively removed by putting a high-speed burr inside to

reach an extended curettage. In the meantime, the defective autogenous bone can be grafted ${ }^{[4,33]}$. In the follow-up imaging after the surgery in this case, the grafted bone seemed to fit the focus well, which suggested that this patient can achieve a good recovery.

\section{Conclusions}

Although $\mathrm{CB}$ is different from GCT and $\mathrm{ABC}$ with respect to the onset characteristics, some of the clinical features and imaging findings overlap. As a consequence, it is critical to device a scheme whereby these 3 diseases can be definitively distinguished to obtain an accurate diagnosis. More attention should be payed to cases where $A B C$ secondary to $C B$ or GCT occurs at rare positions. The most effective approach currently available for this purpose is histological examinations after incision biopsy or excision biopsy.

\section{Abbreviations}

$\mathrm{CB}$ : Chondroblastoma; $\mathrm{ABC}$ : aneurysmal bone cyst; $\mathrm{CT}$ : computed tomography; MRI: magnetic resonance imaging; GCT: giant cell tumor.

\section{Declarations}

\section{Ethics approval and consent to participate}

The collection of patient's clinical data was approved by the Ethics Committee of the General Hospital of Ningxia Medical University and performed in accordance with the Declaration of Helsinki.

\section{Consent for publication}


Written informed consent was obtained from the patient for publication of this report and accompanying images. A copy of this written consent is available for review by the Editor-in Chief of this journal.

\section{Availability of data and materials}

The datasets used and/or analyzed during the current study are available from the corresponding author on reasonable request.

\section{Competing interests}

The authors declare that they have no competing interests.

\section{Funding}

No funding.

\section{Authors' contributions}

J. Zheng made substantial contributions to analysis and interpretation of data and was involved in drafting the manuscript and revising it critically for important content. N. Niu made hudge contributions to conception and design. X. Zhang, C. Liu and X. Zhu collected the patient data. J. Wang performed the histological examination. J. Shi gave final approval of the version to be published and agreed to be responsible for all aspects of the work. All authors read and approved the final manuscript.

\section{Acknowledgements}

Not applicable.

\section{References}

1. Baumhoer D, Harder D., Ameline B, Dawson H., Kollar A. Metastasizing chondroblastoma: a rare bone tumor no longer supported by the WHO classification. Skeletal Radiol. 2020; doi:10.1007/s00256020-03525-6.

2. Liu Q, He HB, Yuan YH, Zeng H, Long F, Tian J, et al. Have the difficulties and complications of surgical treatment for chondroblastoma of the adjoining knee joint been overestimated?. J Bone Oncol, 2019; doi:10.1016/j.jbo.

3. Xu H, Nugent D, Monforte HL, Binitie OT, Ding Y, Letson GD, et al. Chondroblastoma of bone in the extremities: a multicenter retrospective study. J Bone Joint Surg Am. 2015; 97: 925-31.

4. Song MZ, Zhang Z, Wu YX, Ma K, Lu M. Primary tumors of the patella. World J Surg Oncol. 2015; 13: 163.

5. Lang Y, Yu Q, Liu YH, Yang LJ. Chondroblastoma of the patella with pathological fracture in an adolescent: a case report. World J Surg Oncol. 2019; 17: 218. 
6. Tan HG, Yan MN, Yue B, Zeng YM, Wang Y. Chondroblastoma of the patella with aneurysmal bone cyst. Orthopedics, 2014; 37: e87-91.

7. Konishi E, Nakashima Y, Mano M, Tomita Y, Kubo T, Araki N, et al. Chondroblastoma of extracraniofacial bones: Clinicopathological analyses of 103 cases. Pathol. Int., 2017; 67: 495-502.

8. Kolodny A. Bone sarcoma: the primary malignant tumors of bone and the giant cell tumor. Surg Gynecol Obstet. 1927; 44(Suppl 1): 1-214.

9. Codman EA. Epiphyseal chondromatous giant cell tumors of the upper end of the humerus. Surg Gynecol Obstet. 1931; 52: 543-8.

10. De Mattos CB, Angsanuntsukh C, Arkader A, Dormans JP. Chondroblastoma and chondromyxoid fibroma. J Am Acad Orthop Surg. 2013; 21: 225-33.

11. Jaffe HL, Lichtenstein L. Benign Chondroblastoma of Bone: A Reinterpretation of the So-Called Calcifying or Chondromatous Giant Cell Tumor. Am. J. Pathol.. 1942; 18: 969-91.

12. Singh J, James SL, Kroon HM, Woertler K, Anderson SE, Jundt G, et al. Tumour and tumour-like lesions of the patella--a multicentre experience. Eur Radiol. 2009; 19: 701-12.

13. Casadei R, Kreshak J, Rinaldi R, Rimondi E, Bianchi G, Alberghini M, et al. Imaging tumors of the patella. Eur J Radiol. 2013; 82: 2140-8.

14. Desnoyers V, Charissoux JL, Aribit F. Aneurysmal bone cyst of the patella. A case report and literature review. Rev Chir Orthop Reparatrice Appar Mot. 2000; 86: 616-20.

15. Laitinen MK, Stevenson JD, Evans S, Abudu A, Sumathi V, Jeys LM, et al. Chondroblastoma in pelvis and extremities-a signle centre study of 177 cases. J Bone Oncol. 2019; 17: 100248.

16. Scotto di Carlo F, Whyte MP, Gianfrancesco F. The two faces of giant cell tumor of bone. Cancer Lett.. 2020; 489: 1-8.

17. Zeng JC, Zhou M, Xu LH, Zhu LF, Yan ZJ, Wu WD, et al. Curettage through a wide cortical window for treatment of a primary aneurysmal bone cyst of the patella. J. Int. Med. Res..2020; 48: 300060520947910.

18. Balke M, Dedy N, Mueller-Huebenthal J, Liem D, Hardes J, Hoeher J. Uncommon cause for anterior knee pain-Aggressive aneurysmal bone cyst of the patella. Sports Med Arthrosc Rehabil Ther Technol. 2010; 2: 9.

19. Xie C, Jeys Lee, James Steven LJ. Radiofrequency ablation of chondroblastoma: Iong-term clinical and imaging outcomes. Eur Radiol. 2015; 25: 1127-34.

20. Montgomery C, Couch C, Emory CL, Nicholas R. Giant Cell Tumor of Bone: Review of Current Literature, Evaluation, and Treatment Options. J Knee Surg. 2019; 32: 331-336.

21. Traoré A, Doukouré B, Sie Essoh JB, Mobiot C, Soumaro K. Primary aneurysmal bone cyst of the patella: a case report. Orthop Traumatol Surg Res. 2011; 97: 221-4.

22. Ozan F, Toker G. Secondary aneurysmal bone cyst of the patella. Acta Orthop Traumatol Turc. 2010; 44: 246-9. 
23. Zekry KM, Yamamoto N, Hayashi K, Takeuchi A, Araki Y, Alkhooly AZA, et al. Surgical treatment of chondroblastoma using extended intralesional curettage with phenol as a local adjuvant. J Orthop Surg (Hong Kong). 2019; 27: 2309499019861031.

24. Balke M, Dedy N, Mueller-Huebenthal J, Liem D, Hardes J., Hoeher J. Uncommon cause for anterior knee pain - Aggressive aneurysmal bone cyst of the patella. Sports Med Arthrosc Rehabil Ther Technol. 2010; 2: 9.

25. Yu GH, Maisel J, Frank R, Pukenas BA, Sebro R, Weber K. Diagnostic utility of fine-needle aspiration cytology of lesions involving bone. Diagn. Cytopathol.. 2017; 45: 608-613.

26. Suh $\mathrm{CH}$, Yun SJ. Diagnostic Outcome of Image-Guided Percutaneous Core-Needle Biopsy of Sclerotic Bone Lesions: A Meta-Analysis. AJR Am J Roentgenol. 2019; 212: 625-631.

27. Hegde V, Burke ZDC, Park HY, Zoller SD, Johansen D, Kelley BV, et al. Is Core Needle Biopsy Reliable in Differentiating Between Aggressive Benign and Malignant Radiolucent Bone Tumors?. Clin. Orthop. Relat. Res.. 2018; 476: 568-577.

28. Li Y, Du Y, Luo TY, Yang HF, Yu JH, Xu XX, et al. Factors influencing diagnostic yield of CT-guided percutaneous core needle biopsy for bone lesions. Clin Radiol. 2014; 69: e43-7.

29. Behjati S, Tarpey PS, Presneau N, Scheipl S, Pillay N, Van Loo P, et al. Distinct H3F3A and H3F3B driver mutations define chondroblastoma and giant cell tumor of bone. Nat. Genet.. 2013; 45: 147982.

30. Venneker S, Szuhai K, Hogendoorn PCW, Bovée JVMG. Mutation-driven epigenetic alterations as a defining hallmark of central cartilaginous tumours, giant cell tumour of bone and chondroblastoma. Virchows Arch.. 2020; 476: 135-146.

31. Amary MF, Berisha F, Mozela R, Gibbons R, Guttridge A, O'Donnell P, et al. The H3F3 K36M mutant antibody is a sensitive and specific marker for the diagnosis of chondroblastoma. Histopathology. 2016; 69: 121-7.

32. Cleven AH, Höcker S, Briaire-de Bruijn I, Szuhai K, Cleton-Jansen AM, Bovée JV. Mutation Analysis of H3F3A and H3F3B as a Diagnostic Tool for Giant Cell Tumor of Bone and Chondroblastoma. Am. J. Surg. Pathol.. 2015; 39: 1576-83.

33. Chen WQ, DiFrancesco LM. Chondroblastoma: An Update. Arch. Pathol. Lab. Med.. 2017; 141: 867871.

\section{Figures}



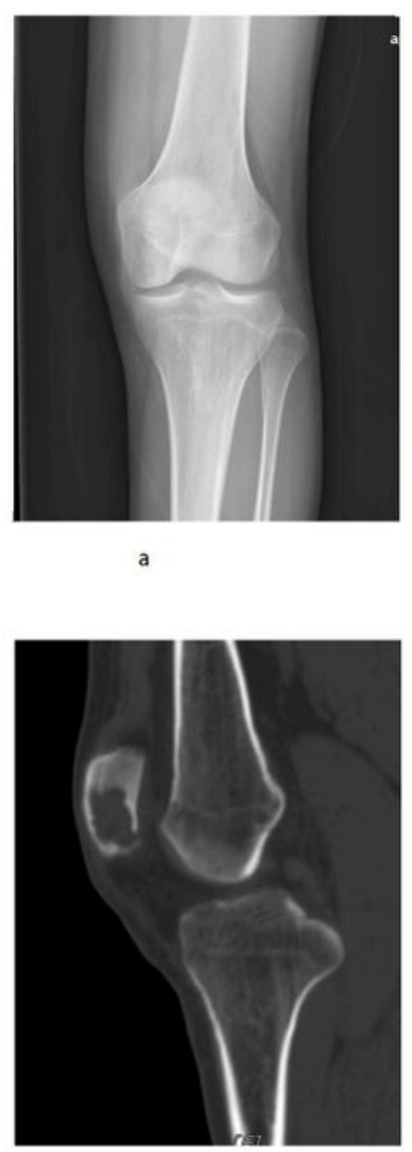

e

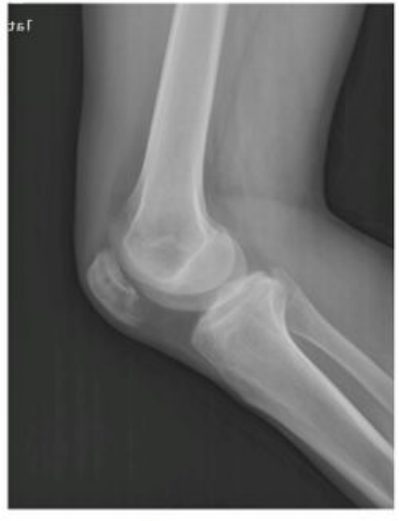

b

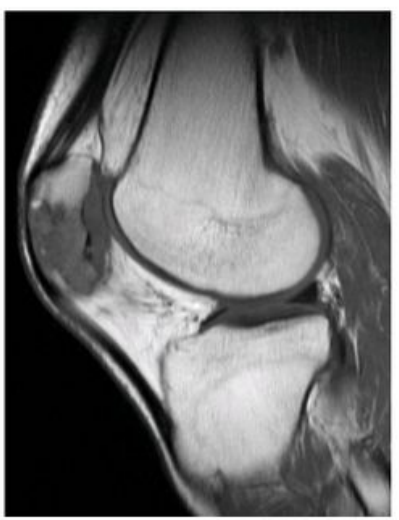

f

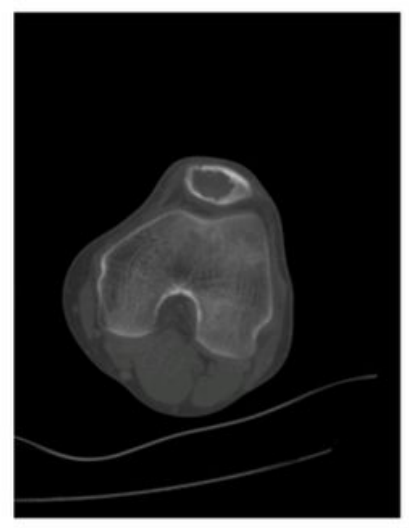

c

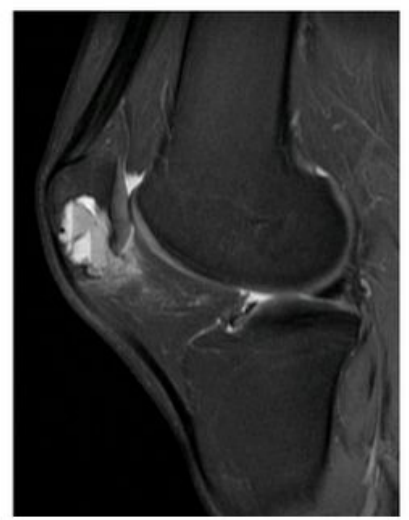

g

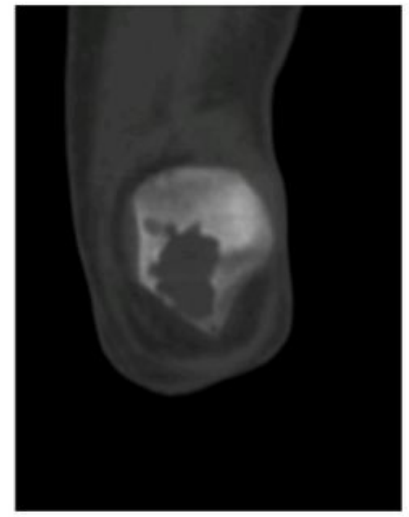

d

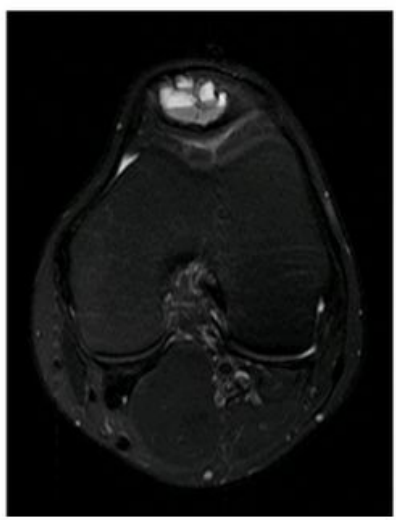

$\mathrm{h}$

\section{Figure 1}

Pre-surgery imaging results $(a, b)$. The radioparent lesion in the patella can be seen in the anteroposterior and lateral X-rays (c, d) The focus with a sharp edge, in a lobulated or worm-eaten shape, was observed in the axial and coronal views of pre-surgery CT. No boundary or internal calcification were noted in the focus (e) At the sagittal view of pre-surgery CT, it can be seen that the focus was connected with the joint cavity at the junction between the inferior patella and the articular surface $(f, g, h)$ In pre-surgery MRI, $f$ was T1-weighted image. The patella focus was hypointensity. $\mathrm{g}$ and $\mathrm{h}$ were T2-weighted images. The focus was hyperintense, and the fluid level was present in it. Therefore, secondary ABC was suggestive. 


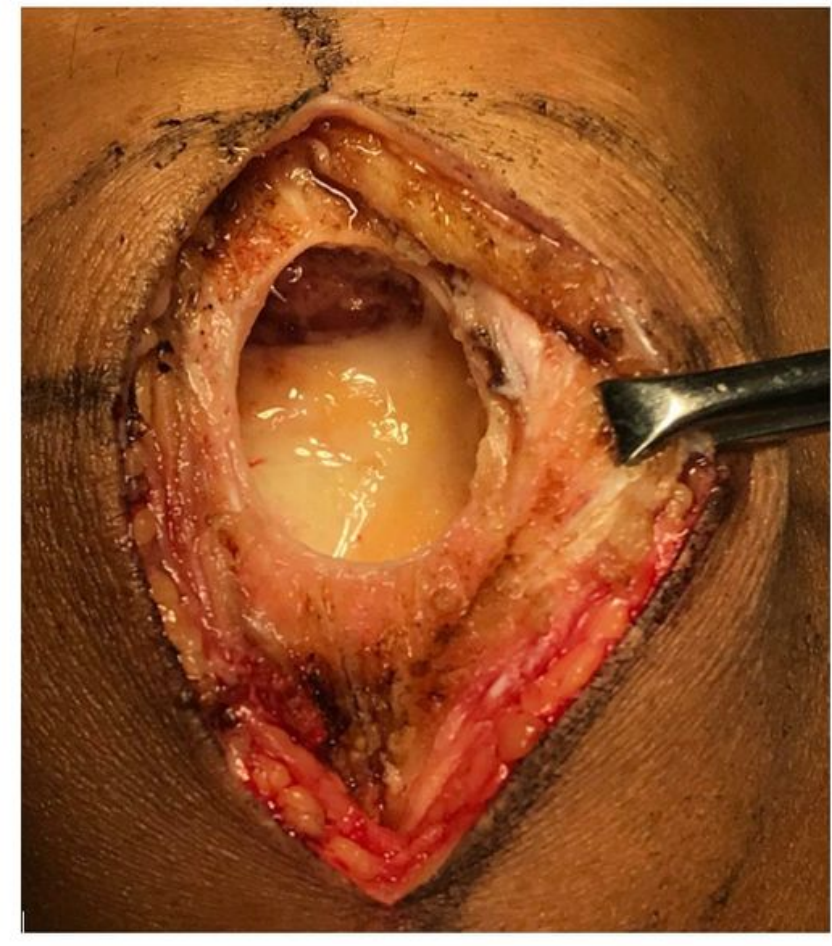

a

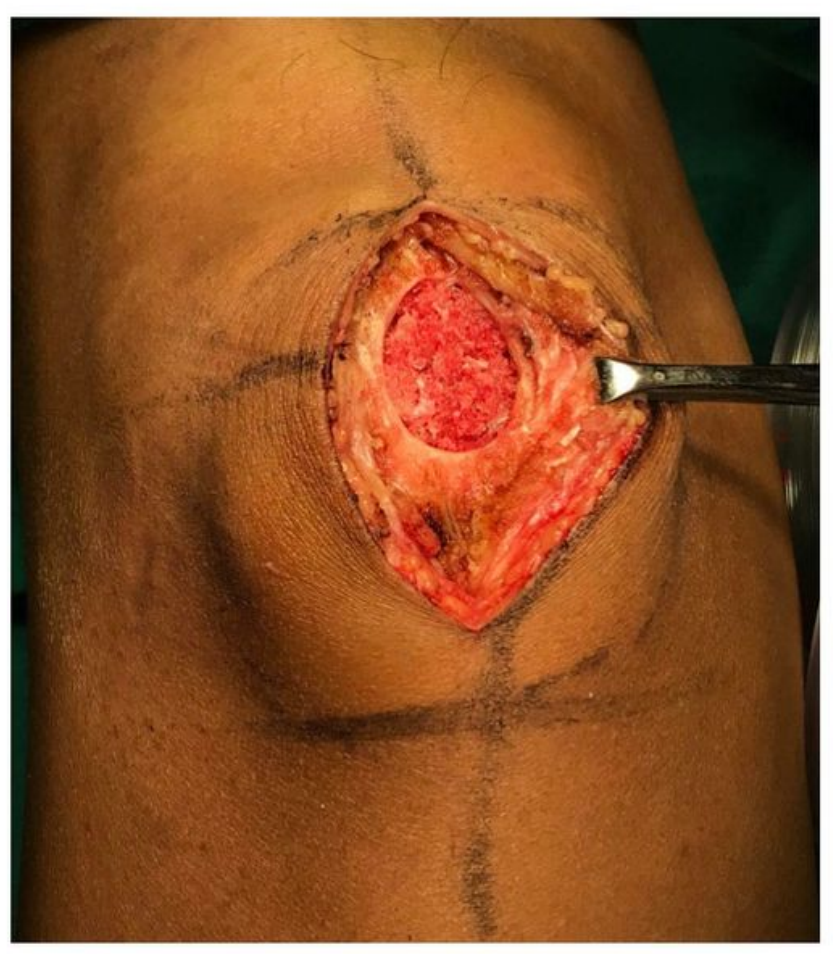

b

\section{Figure 2}

Figures before and after bone grafting at the focus during the surgery (a) The focus was debrided through the oval cortical window on the anterior patella. It can be seen here that multiple partitions of the focus were totally removed and the cartilage surface of the patella was complete (b) Autologous bone was grafted at the focus and filled the latter well.

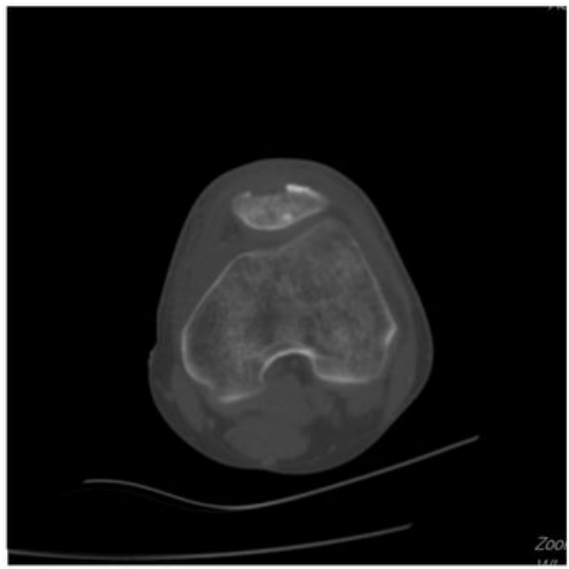

a

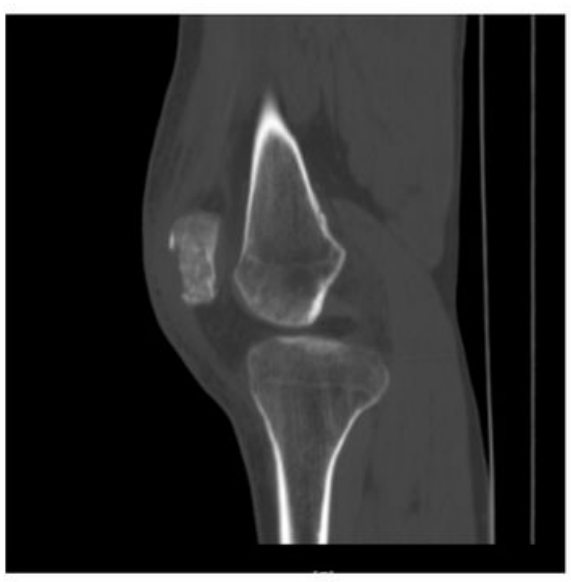

b

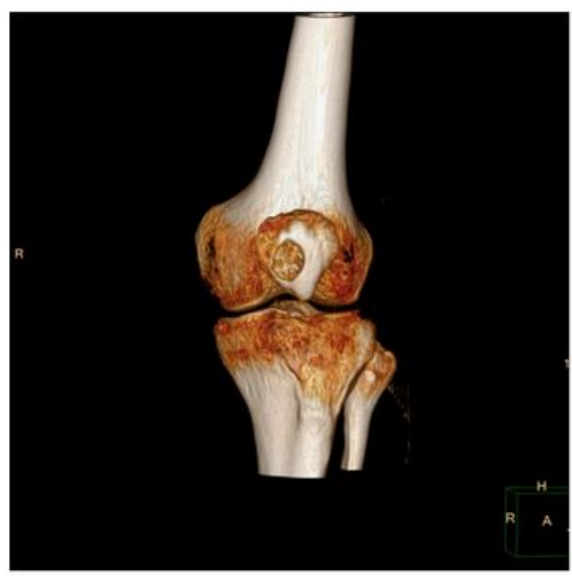

C

Figure 3 
Post-surgery imaging data $(a, b, c)$ The fenestration for the focus was found to be reasonable in the postsurgery reconstructed, axial and sagittal CT, and patella-reconstructed CT. The bone-grafting effect was found to be satisfactory on the axial and sagittal CT.

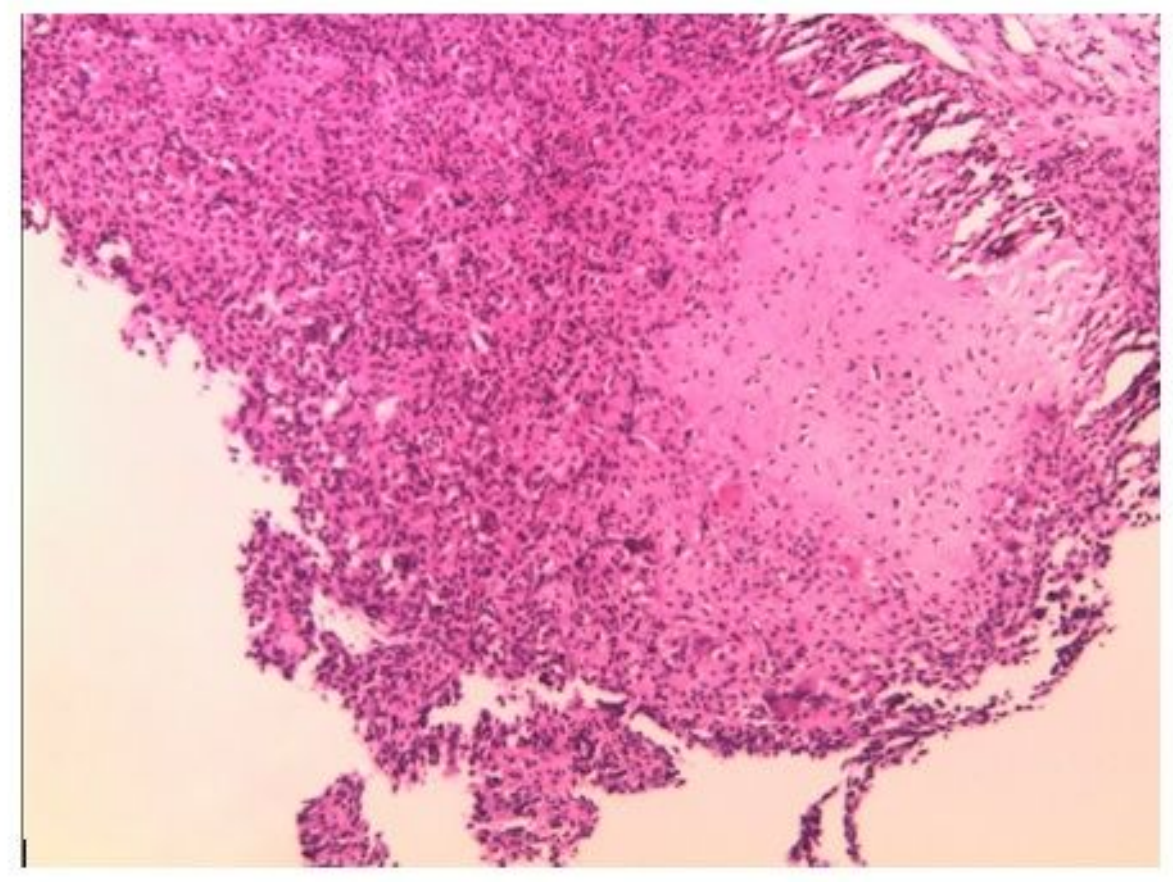

a

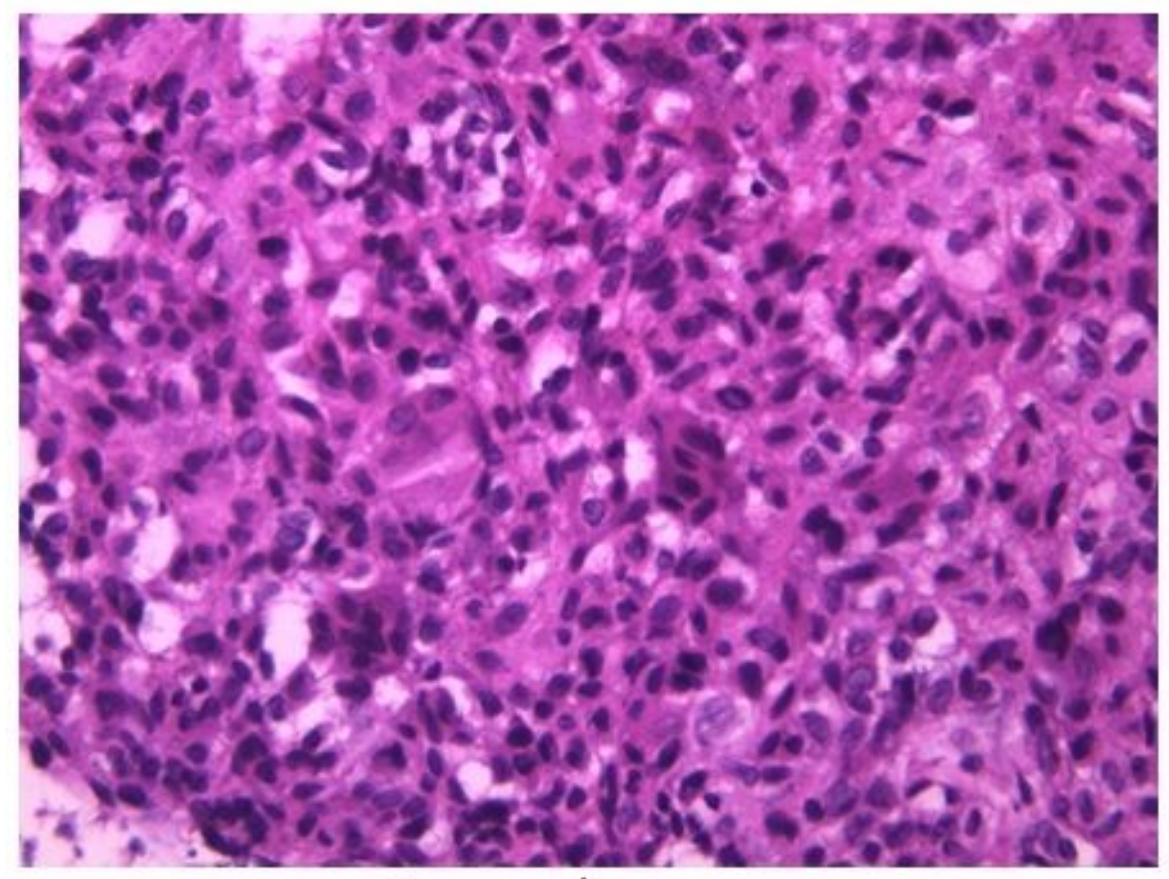

b

Figure 4

Pathological outcomes of the surgery (a) The tumor was composed of confluent proliferating cells in round and oval shapes, and osteoclast-like multi-nucleated giant cells were scattered throughout the 
tumor. x100 (b) Under high-power microscope, the tumor cells showed up as round cells with a clear boundary, while the cytoplasm was light red in color or transparent with nuclear grooves. Pink cartilage matrix was observed in the tumor cells. $x 400$ 